\title{
On a Class of Smooth Preferences*
}

\author{
Andrea Attar ${ }^{\dagger} \quad$ Thomas Mariotti ${ }^{\ddagger}$ François Salanié ${ }^{\S}$
}

October 13, 2017

\begin{abstract}
We construct a complete space of smooth strictly convex preference relations defined over physical commodities and monetary transfers. This construction extends the classical one by assuming that preferences are monotone in transfers, but not necessarily in all commodities. We thereby provide a natural framework to perform genericity analyses in situations involving inventory costs or decisions under risk. The space of preferences we construct is contractible, which allows for a natural aggregation procedure in collective decision situations.
\end{abstract}

Keywords: Smooth Preferences, Nonmonotonicity, Collective Choice. JEL Classification: C60, D11.

\footnotetext{
${ }^{*}$ We thank the editor and two anonymous referees for very thoughtful and detailed comments. We also thank Michel Le Breton and Jérôme Renault for extremely valuable feedback.

†Università degli Studi di Roma "Tor Vergata," Roma, Italy, and Toulouse School of Economics, CNRS, University of Toulouse Capitole, Toulouse, France.

¥Toulouse School of Economics, CNRS, University of Toulouse Capitole, Toulouse, France.

$\S$ Toulouse School of Economics, INRA, University of Toulouse Capitole, Toulouse, France.
} 


\section{Introduction}

Applications of classical demand theory tend to treat each commodity either as a "good" or as a "bad." This implies that we can, if necessary, redefine the commodities so as to treat all of them as goods: ${ }^{1}$ thus leisure time is the flip side of hours worked, and clean air or water is the flip side of pollution. Technically, this allows us to focus on preferences that are monotone in each commodity. ${ }^{2}$

However, even in this sense, the monotonicity of preferences is not always guaranteed. For instance, if a consumer or a firm cannot freely dispose of a stock of unwanted commodities, they will typically have to bear inventory costs for holding this stock; for instance, they may need to acquire a new storage facility. In the presence of such costs or, more generally, when the individual's objective function is an indirect utility function derived under technological constraints, there are situations where, beyond a certain point, more of a commodity actually decreases the individual's utility or profit. As a result, we cannot unambiguously classify such a commodity as a good or a bad, as this ultimately depends on how much of it the individual is endowed with.

A prominent example of nonmonotonity arises in financial economics, when we consider investors' preferences over portfolios of assets. While larger holdings of cash or risk-free assets are always desirable from an investor's viewpoint, this is not so for risky securities whenever he is risk-averse: indeed, moderate holdings of a risky security are desirable for hedging or speculative purposes, but too large a risk exposure is not. Another natural example of nonmonotone preferences arises in the modeling of groups such as households, families, firms, unions, clubs, or, more generally, in situations where individuals are expected to make private contributions to collective goods or activities. In this type of environments, it is standard to assume that each individual benefits on the whole from making moderate contributions, but eventually experiences overwhelming costs whenever his contributions exceed a threshold.

In these contexts, the classical construction of a complete space of smooth strictly convex preference relations (Mas-Colell (1985, Chapter 2)) has to be amended. We propose such a canonical model of preferences in which nonmonotonicity is allowed for certain commodities, but there is one commodity that is always desirable. Monetary transfers to the consumer

\footnotetext{
${ }^{1}$ See, for instance, Varian (1992, Chapter 7) or Mas-Colell, Whinston, and Green (1995, Chapter 3).

${ }^{2}$ To be fair, much of classical demand theory can be developed by relying on the weaker local-nonsatiation assumption, but monotonicity appears to be the rule in applications. The implications of nonmonotone preferences for the existence and efficiency of competitive equilibria have been examined in Polemarchakis and Siconolfi (1993), among others. In their approach, however, they take consumers' preferences as given and do not develop a framework for genericity analysis.
} 
yield a straightforward example of such a commodity, and we will stick to this interpretation in most of the paper; this is in particular relevant for principal-agent models where transfers are allowed. But other examples of such a commodity are easy to find: think of leisure time in a household, or the practice of a shared activity in a club. Our first contribution is thus to extend the classical construction to this class of environments, giving rise to a topologically complete space of smooth strictly convex nonmonotone preference relations. This construction enables new genericity analyses: we may, for instance, use it to check the robustness of results obtained in portfolio-choice theory under the usual CARA-normal specification, without assuming that the investor's primitive preferences over state-contingent consumption have an expected-utility representation. ${ }^{3}$

An important property of this space of preferences is that it is contractible, that is, it can be continuously deformed into a single preference relation. Key to this result is a compensation principle that states that an individual can be compensated for holding any stock of commodities through appropriate transfers of the last, uniformly desirable commodity. In line with Chichilnisky and Heal (1983), contractibility can intuitively be interpreted as a "topological unanimity" condition; in particular, profiles of such preferences can be continuously deformed into unanimous profiles. This suggests that our space of preferences admits nice aggregation properties. We show that this is indeed the case: there exists a collective choice rule over profiles of such preferences that is continuous, anonymous, and respects unanimity. Although this finding is in line with the results of the literature on topological social choice initiated by Chichilnisky (1980), it is not a direct consequence of known results; instead, it is a consequence of the particular contraction we construct on the space of preferences.

The paper is organized as follows. Section 2 describes a space of basic preferences. Section 3 introduces our compensation principle and a space of normalized preferences. Section 4 provides the construction of our complete space of preferences. Section 5 draws the implications of our analysis for collective choice.

\section{Basic Preferences}

In this section, we introduce a space $\mathbf{P}$ of basic preferences. There are $\ell+1$ commodities, the last of which represents transfers to the consumer. We denote by $q$ a vector of the first

\footnotetext{
${ }^{3}$ Notice in that respect that, when the state space is infinite, conducting a genericity analysis in the finite-dimensional space of portfolio choices is mathematically much simpler than doing so in the infinitedimensional space of state-contingent consumption choices.
} 
$\ell$ commodities, by $t$ a scalar amount of transfers, and by $0_{\ell}$ the null vector in $\mathbb{R}^{\ell}$. We shall consider regular preference relations $\succeq$ over an open subset $V$ of $\mathbb{R}^{\ell+1}$. We require that $V$ contain the no-trade point $\left(0_{\ell}, 0\right)$, which corresponds to the consumer's endowment point, that it be convex with a nonempty interior, and that it be comprehensive with respect to transfers, in the sense that, if $(q, t) \in V$ and $t^{\prime}>t$, then $\left(q, t^{\prime}\right) \in V$; thus $V$ is unbounded from above in the direction of transfers. We let $Q \equiv \operatorname{proj}_{\mathbb{R}^{\ell}} V$ and, for each $q \in Q$, we let $\underline{t}(q) \equiv \inf \{t \in \mathbb{R}:(q, t) \in V\}$; notice that, as $V$ is convex, $\underline{t}\left(q^{\prime}\right)=-\infty$ for all $q^{\prime} \in Q$ if $\underline{t}(q)=-\infty$ for some $q \in Q$. Figure 1 below illustrates these assumptions.

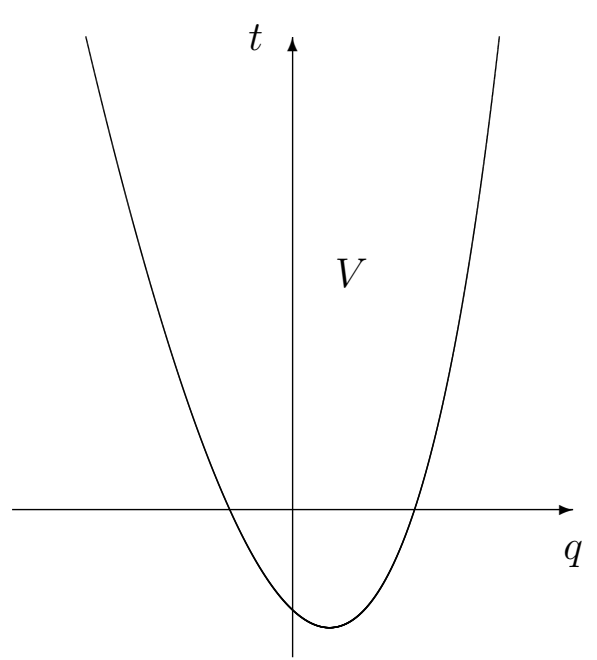

Figure 1.a An admissible domain $V$. Figure 1.b A nonadmissible domain $V$.

We impose the following axioms on $\succeq$ :

A1 $\succeq$ is closed relative to $V \times V$.

A2 $\succeq$ is strictly monotone in transfers: if $(q, t) \in V$ and $t^{\prime}>t$, then $\left(q, t^{\prime}\right) \succ(q, t)$.

A3 $\succeq$ is convex: if $(q, t) \succeq\left(q^{\prime}, t^{\prime}\right)$ and $\lambda \in[0,1]$, then $\lambda(q, t)+(1-\lambda)\left(q^{\prime}, t^{\prime}\right) \succeq\left(q^{\prime}, t^{\prime}\right)$.

A4 $\succeq$ has closed upper contour sets relative to $\mathbb{R}^{\ell+1}$.

A5 $\succeq$ has a boundary in $V \times V$ that is a $C^{2}$ manifold.

A1 and A3 are standard. A2 requires that preferences be strictly monotone in transfers. This generalizes the standard axiom that requires that preferences be strictly monotone in all the commodities; if we were to impose this stronger requirement, we would obtain the class of 
preferences studied in Mas-Colell (1985, Chapter 2). A4 describes the boundary behavior of preferences. Although its role is mainly technical, A4 also has an economic interpretation; for instance, in case $V=\prod_{l=1}^{\ell}\left(q_{l}^{-}, q_{l}^{+}\right) \times\left(q_{\ell+1}^{-}, \infty\right)$, for some (possibly infinite) negative and positive numbers $q_{l}^{-}$and $q_{l}^{+}$, A4 expresses an indispensability property: subsistence ceases to be possible if the consumption or sale of commodity $l=1, \ldots, \ell$ attain some thresholds $q_{l}^{+}$and $\left|q_{l}^{-}\right|$, and there is a maximum debt limit $\left|q_{\ell+1}^{-}\right|$at which the consumer must declare bankruptcy. ${ }^{4}$ Finally, A5 requires that preferences be sufficiently regular.

Example Assume that a risk-averse investor with constant absolute risk-aversion $\alpha$ can invest in $\ell$ risky assets with payoffs that are jointly normally distributed with mean vector $a$ and covariance matrix $\Gamma$, as well as in a risk-free asset with payoff 1 . Then his preferences over portfolios of risky and risk-free assets $(q, t) \in V \equiv \mathbb{R}^{\ell+1}$ are represented by

$$
u(q, t)=q^{\top} a-\frac{\alpha}{2} q^{\top} \Gamma q+t
$$

and satisfy A1-A5. Notice the nonmonotonity in $q$, reflecting that the investor does not want to hold an excessively risky asset position.

Our first task is to characterize the space $\mathbf{P}$ of preferences $\succeq$ that satisfy A1-A5. The following notation will be useful. Let $U_{(q, t)}$ and $L_{(q, t)}$ be the upper and lower contour sets of $(q, t)$ for $\succeq$, and let $I_{(q, t)} \equiv U_{(q, t)} \cap L_{(q, t)}$ be the indifference set of $(q, t)$ for $\succeq$. Observe by A2 that $U_{(q, t)}$ is comprehensive with respect to transfers, just as $V$. Also denote by cl and $\partial$ the closure and boundary operators relative to $V$ or $V \times V$, depending on the context. We start with two technical lemmas.

Lemma 1 If $\succeq$ satisfies A1-A2, then, for each $(q, t) \in V, U_{(q, t)}$ has a nonempty interior relative to $\mathbb{R}^{\ell+1}$ and $I_{(q, t)}=\partial U_{(q, t)}$.

Proof. To prove the first claim, observe that, as $\succeq$ is closed relative to $V \times V$ by A1, $V \backslash L_{(q, t)}$ is open relative to $V$, and thus relative to $\mathbb{R}^{\ell+1}$ as $V$ is an open subset of $\mathbb{R}^{\ell+1}$. Hence, because $V \backslash L_{(q, t)}$ is nonempty by A2, $U_{(q, t)} \supset V \backslash L_{(q, t)}$ has a nonempty interior relative to $\mathbb{R}^{\ell+1}$. To prove the second claim, observe that, by $\mathrm{A} 1$ again, $U_{(q, t)}$ and $L_{(q, t)}$ are closed relative to $V$. Therefore, we have

$$
\partial U_{(q, t)} \equiv \operatorname{cl}\left(U_{(q, t)}\right) \cap \operatorname{cl}\left(V \backslash U_{(q, t)}\right)=U_{(q, t)} \cap \operatorname{cl}\left(V \backslash U_{(q, t)}\right) \subset U_{(q, t)} \cap L_{(q, t)}=I_{(q, t)}
$$

\footnotetext{
${ }^{4}$ It should be noted that $\mathrm{A} 4$ does not follow from $\mathrm{A} 1$ if $V$ is a proper subset of $\mathbb{R}^{\ell+1}$, for then $\succeq$ can be closed in the open set $V \times V$ in the relative topology, though its upper contour sets are adherent to the boundary of $V$ in $\mathbb{R}^{\ell+1}$ and are thus not closed relative to $\mathbb{R}^{\ell+1}$.
} 
The reverse inclusion is satisfied if $I_{(q, t)} \subset \mathrm{cl}\left(V \backslash U_{(q, t)}\right)$, which is obviously true because, for each $\left(q^{\prime}, t^{\prime}\right) \in I_{(q, t)},\left(q^{\prime}, t^{\prime}-\varepsilon\right) \in V$ for any small enough $\varepsilon>0$ by openness of $V$, and $\left(q^{\prime}, t^{\prime}\right) \succ\left(q^{\prime}, t^{\prime}-\varepsilon\right)$ for any such $\varepsilon$ by A2. The result follows.

Lemma 2 If $\succeq$ satisfies $A 1-A 4$, then, for each $(q, t) \in V, I_{(q, t)}$ is connected.

Proof. By Lemma 1, $I_{(q, t)}=\partial U_{(q, t)}$, so that we can focus on the topological properties of $U_{(q, t)}$. By A3-A4, $U_{(q, t)}$ is a closed convex subset of $\mathbb{R}^{\ell+1}$; moreover, $U_{(q, t)}$ has a nonempty interior by Lemma 1. Hence two cases may arise (Klee (1953, III.1.6)).

Case 1 Either the asymptotic cone $\mathbf{A} U_{(q, t)} \equiv\left\{x \in \mathbb{R}^{\ell+1}:\left(q^{\prime}, t^{\prime}\right)+\lambda x \in U_{(q, t)}\right.$ for all $\left.\left(q^{\prime}, t^{\prime}, \lambda\right) \in U_{(q, t)} \times \mathbb{R}_{+}\right\}$of $U_{(q, t)}$ is not a linear subspace. Then $U_{(q, t)}$ is homeomorphic with $\mathbb{R}^{\ell} \times[0,1)$ and $\partial U_{(q, t)}$ with $\mathbb{R}^{\ell}$. In particular, $\partial U_{(q, t)}$ is connected.

Case 2 Or the asymptotic cone $\mathbf{A} U_{(q, t)}$ of $U_{(q, t)}$ is an $\ell+1-k$-dimensional linear subspace, for some integer $k \leq \ell+1$. Because $U_{(q, t)}$ is comprehensive with respect to transfers, we must have $k \leq \ell$ and $\left(0_{\ell}, 1\right) \in \mathbf{A} U_{(q, t)}$. As $\mathbf{A} U_{(q, t)}$ is a linear subspace, it follows that $\left(0_{\ell},-1\right) \in \mathbf{A} U_{(q, t)}$. This implies that $\left(q, t^{\prime}\right) \succeq(q, t)$ for all $t^{\prime}<t$ such that $\left(q, t^{\prime}\right) \in V$, which is ruled out by A2. This case is thus impossible. The result follows.

Let $\mathbf{U}$ be the space of quasiconcave $C^{2}$ functions $u: V \rightarrow \mathbb{R}$ such that $\partial u / \partial t>0$ over $V$ and $u^{-1}([v, \infty))$ is closed in $\mathbb{R}^{\ell+1}$ for all $v \in \mathbb{R}$. Lemmas $1-2$ then imply the following representation result.

Proposition $\mathbf{1} \succeq$ satisfies A1-A5 if and only if it admits a utility function $u \in \mathbf{U}$.

Proof. (Direct part) Suppose that $\succeq$ is representable by $u$. Then $\succeq$ trivially satisfies A1-A3. Next, as $u^{-1}([v, \infty))$ is closed in $\mathbb{R}^{\ell+1}$ for all $v \in \mathbb{R}, \succeq$ satisfies A4. Finally, because $u$ clearly has no critical point, that is, $\partial u \neq 0$ over $V$, it follows as in Mas-Colell (1985, Proposition 2.3.5) that $\succeq$ satisfies A5.

(Indirect part) By A2, $\succeq$ is locally nonsatiated, and by A5, $\partial \succeq$ is a $C^{2}$ manifold in $V \times V$. Hence $\succeq$ is of class $C^{2}$ (Mas-Colell (1985, Definition 2.3.4)). Moreover, by Lemmas 1-2, $\succeq$ has connected indifference sets $I_{(q, t)}$. Hence it admits a $C^{2}$ utility function $u$ over $V$ with no critical point (Mas-Colell (1985, Proposition 2.3.9)). That $u$ is quasiconcave follows from A3. To show that $\partial u / \partial t>0$ over $V$, observe first from A2 that $\partial u / \partial t \geq 0$ over $V$. Now, suppose, by way of contradiction, that $(\partial u / \partial t)(q, t)=0$ for some $(q, t) \in V$. Then $(\partial u / \partial q)(q, t) \neq 0_{\ell}$ as $u$ has no critical point. Thus the hyperplane through $(q, t)$ orthogonal to $\partial u(q, t)$ that 
supports the convex set $U_{(q, t)}$ is vertical. It follows that the strict upper contour set of $(q, t)$ for $\succeq, U_{(q, t)} \backslash L_{(q, t)}$, strictly lies on one side or the other of this hyperplane. This does not include the half line $\left\{\left(q, t^{\prime}\right) \in V: t^{\prime}>t\right\}$, which contradicts A2. Hence $\partial u / \partial t>0$ over $V$, as claimed. Finally, that $u^{-1}([v, \infty))$ is closed in $\mathbb{R}^{\ell+1}$ for all $v \in \mathbb{R}$ is a direct consequence of A4. Hence the result.

Proposition 1 states that any preference relation in $\mathbf{P}$ can be represented by some function in $\mathbf{U}$ and, conversely, that any function in $\mathbf{U}$ represents a preference relation in $\mathbf{P}$. For each $u \in \mathbf{U}$, let $P(u) \subset V \times V$ be the preference relation represented by $u$. In line with Mas-Colell (1985, Chapter 2, Section 4), a topology over $\mathbf{P}$ can be constructed as follows. Note that $\mathbf{U}$ is a subspace of $C^{2}(V)$, the Polish space of real-valued $C^{2}$ functions over $V$ endowed with the topology of uniform convergence over compact subsets of $V$ of functions and of their derivatives up to the order 2 (Mas-Colell (1985, Chapter 1, K.1.2)). Then we endow $\mathbf{P}$ with the identification topology from $P$; that is, we let $O$ be open in $\mathbf{P}$ if $P^{-1}(O)$ is open in $\mathbf{U}$. Note that $P$ is not one-to-one; however, we can show as in Mas-Colell (1985, Chapter 2, Proposition 2.4.2) that $P$ is open, which implies that a sequence $\left(\succeq_{n}\right)_{n \in \mathbb{N}}$ converges to $\succeq$ in $\mathbf{P}$ if and only if there exists a sequence of representations $\left(u_{n}\right)_{n \in \mathbb{N}}$ for the preferences $\left(\succeq_{n}\right)_{n \in \mathbb{N}}$ that converges in $\mathbf{U}$ to a representation $u$ of $\succeq$.

\section{$3 \quad$ Normalized Preferences}

In this section, we introduce a subspace $\mathbf{P}_{v}$ of $\mathbf{P}$, the elements of which admit convenient normalized representations. To this end, we add a further restriction on preferences in the form of the following axiom:

A6 For all $(q, t) \in V$ and $q^{\prime} \in Q$, there exists $t^{\prime}$ such that $\left(q^{\prime}, t^{\prime}\right) \in V$ and $\left(q^{\prime}, t^{\prime}\right) \succeq(q, t)$.

A6 expresses a compensation principle: using appropriate transfers, the consumer can be compensated for holding any feasible amount of the first $\ell$ commodities. The following lemma shows that exact compensation is then possible up to any utility level.

Lemma 3 If $\succeq$ satisfies $A 1-A 4$ and $A 6$, then, for all $(q, t) \in V$ and $q^{\prime} \in Q$, there exists $t^{\prime}$ such that $\left(q^{\prime}, t^{\prime}\right) \in V$ and $\left(q^{\prime}, t^{\prime}\right) \sim(q, t)$.

Proof. Suppose, by way of contradiction, that the result does not hold for some $(q, t) \in V$ and $q^{\prime} \in Q$. Then, by A1-A2 and A6, $\left(q^{\prime}, t^{\prime}\right) \in U_{(q, t)}$ for all $t^{\prime}$ such that $\left(q^{\prime}, t^{\prime}\right) \in V$. We distinguish two cases. First, if $\underline{t}\left(q^{\prime}\right) \in \mathbb{R}$, we have $\left(q^{\prime}, t^{\prime}\right) \in U_{(q, t)}$ for all $t^{\prime}>\underline{t}\left(q^{\prime}\right)$ but 
$\left(q^{\prime}, \underline{t}\left(q^{\prime}\right)\right) \notin V$ as $V$ is open, and thus $\left(q^{\prime}, \underline{t}\left(q^{\prime}\right)\right) \notin U_{(q, t)}$, which contradicts A4. Second, if $\underline{t}\left(q^{\prime}\right)=-\infty$, we have $\left(q^{\prime}, t^{\prime}\right) \in U_{(q, t)}$ for all $t^{\prime} \in \mathbb{R}$; that is, $U_{(q, t)}$ contains a vertical line. Then

$$
\left(1+\frac{1}{t^{\prime}}\right)(q, t)-\frac{1}{t^{\prime}}\left(q^{\prime}, t^{\prime}\right) \in U_{(q, t)}
$$

for all $t^{\prime}<-1$ by A3. Letting $t^{\prime}$ go to $-\infty$, we get by A1 that $(q, t-1) \in U_{(q, t)}$, which contradicts A2. The result follows.

The geometrical interpretation is that any vertical line that intersects $V$ must intersect all the indifference sets of $\succeq$, see Figure 2 below. That is, the indifference sets of $\succeq$ do not admit vertical asymptotes, except perhaps at the boundary of $V$. This property plays a role analogous, in our setup with possibly nonmonotone preferences, to the standard property that the indifference sets of strictly monotone preferences defined over the interior of the positive orthant must intersect any ray in the latter that emanates from the origin.

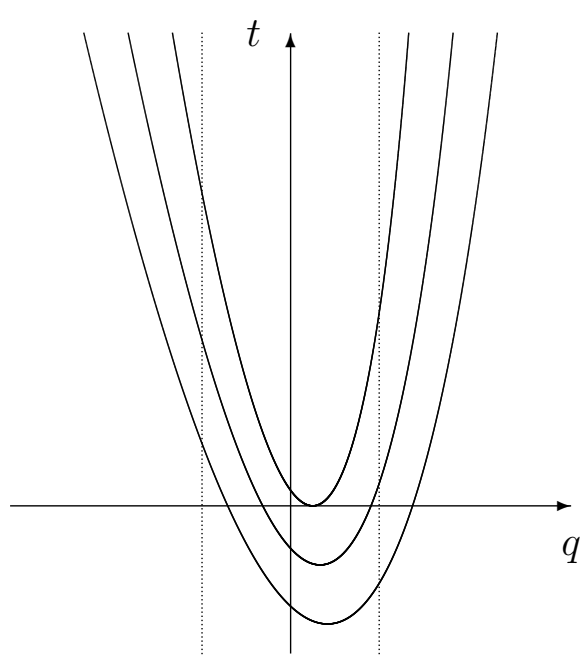

Figure 2.a A6 is satisfied.

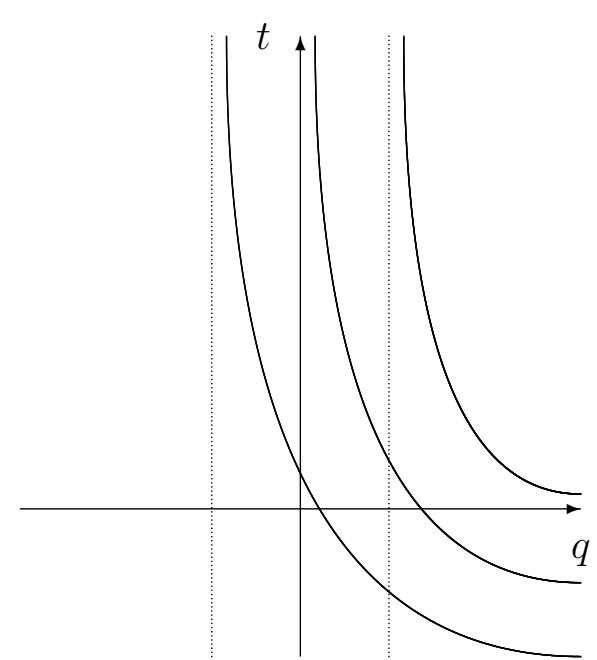

Figure 2.b A6 is violated.

Our task in this section is to characterize the space $\mathbf{P}_{v}$ of preferences $\succeq$ that satisfy A1-A6. Given Lemma 3, it will be convenient to work with a space of normalized utility functions, defined as $\mathbf{U}_{v} \equiv\left\{u \in \mathbf{U}\right.$ : range $u(q, \cdot)=$ range $u\left(0_{\ell}, \cdot\right)$ for all $q \in Q$ and $u\left(0_{\ell}, t\right)=$ $t$ for all $t$ such that $\left.\left(0_{\ell}, t\right) \in V\right\}$. It should be noted that the normalization along the vertical axis satisfied by the utility functions in $\mathbf{U}_{v}$ differs from the standard radial one (Wold and Juréen (1953), Kannai (1970)), reflecting that preferences are monotone in transfers, but not necessarily in the other commodities. We have the following characterization result. 
Proposition $\mathbf{2} \mathbf{U}_{v}$ is homeomorphic with $\mathbf{P}_{v}$ under the natural map $P$.

Proof. As a preliminary remark, let us observe that, for each $u \in \mathbf{U}_{v}, P(u)$ satisfies A1-A5 by Proposition 1 ; moreover, the property that range $u(q, \cdot)=\operatorname{range} u\left(0_{\ell}, \cdot\right)$ for all $q \in Q$ implies that $P(u)$ satisfies the property stated in Lemma 3, and thus, a fortiori, A6. Thus $P(u) \in \mathbf{P}_{v}$ for all $u \in \mathbf{U}_{v}$. We must prove that the mapping $P_{\mid \mathbf{U}_{v}}: \mathbf{U}_{v} \rightarrow \mathbf{P}_{v}: u \mapsto P(u)$ is one-to-one, onto, continuous, and open.

(One-to-one) Let $u$ and $u^{\prime}$ in $\mathbf{U}_{v}$ such that $P(u)=P\left(u^{\prime}\right)$. Then $u=\xi \circ u^{\prime}$, where $\xi: u^{\prime}(V) \rightarrow \mathbb{R}$ is $C^{2}$, increasing, and regular, that is, $\partial \xi>0$ over $u^{\prime}(V)$ (Mas-Colell (1985, Proposition 2.3.11)). This implies that, for each $v \in u^{\prime}(V), \xi(v)=\xi\left(u^{\prime}\left(0_{\ell}, v\right)\right)=u\left(0_{\ell}, v\right)=$ $v$, so that $u=u^{\prime}$.

(Onto) Let $\succeq \in \mathbf{P}_{v}$. From Proposition 1, there exists some $u \in \mathbf{U}$ such that $\succeq=$ $P(u)$. As range $u(q, \cdot)=$ range $u\left(0_{\ell}, \cdot\right)$ for all $q \in Q$, we can implicitly define $u^{\prime}: V \rightarrow \mathbb{R}$ by $u(q, t)=u\left(0_{\ell}, u^{\prime}(q, t)\right)$. We clearly have $P\left(u^{\prime}\right)=\succeq$. We now check that $u^{\prime} \in \mathbf{U}_{v}$. That $u^{\prime}$ is quasiconcave follows from the fact that $\left\{(q, t) \in V: u^{\prime}(q, t) \geq v\right\}=\{(q, t) \in$ $\left.V: u(q, t) \geq u\left(0_{\ell}, v\right)\right\}$ for all $v$ such that $\left(0_{\ell}, v\right) \in V$. This observation also implies that $\left(u^{\prime}\right)^{-1}([v, \infty))$ is closed in $\mathbb{R}^{\ell+1}$ for any such $v$. That $u^{\prime}$ is $C^{2}$ follows from the implicit function theorem along with the fact that $\partial u / \partial t>0$ over $V$. We then have $(\partial u / \partial t)(q, t)=$ $(\partial u / \partial t)\left(0_{\ell}, u^{\prime}(q, t)\right)\left(\partial u^{\prime} / \partial t\right)(q, t)$, which in turn implies that $\partial u^{\prime} / \partial t>0$ over $V$. We also obtain that range $u^{\prime}(q, \cdot)=u^{-1}\left(0_{\ell}\right.$, range $\left.u(q, \cdot)\right)=u^{-1}\left(0_{\ell}\right.$, range $\left.u\left(0_{\ell}, \cdot\right)\right)=\operatorname{range} u^{\prime}\left(0_{\ell}, \cdot\right)$ for all $q \in Q$, as desired. Last, by construction, $u\left(0_{\ell}, t\right)=u\left(0, u^{\prime}\left(0_{\ell}, t\right)\right)$ for all $t$ such that $\left(0_{\ell}, t\right) \in V$, so that, by A2, $u^{\prime}\left(0_{\ell}, t\right)=t$ for any such $t$. Thus $u^{\prime} \in \mathbf{U}_{v}$, as claimed.

(Continuous) This follows from the definition of the topology of $\mathbf{P}$.

(Open) Mimic the proof of Mas-Colell (1985, Proposition 2.4.2)). Hence the result.

\section{Differentiably Strictly Convex Preferences}

We are now ready to complete the construction of the complete and contractible space of preferences announced in the introduction. Preferences in $\mathbf{P}_{v}$ are not necessarily strictly convex. We add this restriction as a further axiom:

A7 $\succeq$ is strictly convex: if $(q, t) \succeq\left(q^{\prime}, t^{\prime}\right),(q, t) \neq\left(q^{\prime}, t^{\prime}\right)$, and $\lambda \in(0,1)$, then $\lambda(q, t)+(1-$ $\lambda)\left(q^{\prime}, t^{\prime}\right) \succ\left(q^{\prime}, t^{\prime}\right)$.

Finally, to obtain a topologically complete space of preferences, we require preferences to be 
nonlinear, even in a local sense. To do so, observe that because a utility function $u \in \mathbf{U}_{v}$ representing a preference $\succeq \in \mathbf{P}_{v}$ has no critical point, the Gaussian curvature $c_{(q, t)}$ of the indifference set $I_{(q, t)}$ at $(q, t)$ is well defined and given by

$$
c_{(q, t)} \equiv \frac{1}{\|\partial u(q, t)\|^{3}}\left|\begin{array}{cc}
-\partial^{2} u(q, t) & \partial u(q, t) \\
-\partial u^{\top}(q, t) & 0
\end{array}\right|
$$

see, for instance, Debreu (1972). The last restriction we impose on preferences is that this curvature nowhere vanishes:

A8 Any point of $V$ is regular for $\succeq$, that is, $c \neq 0$ over $V$.

Preferences that satisfy A7-A8 are said to be differentiably strictly convex (Mas-Colell (1985, Definition 2.6.1)).

We can now define our fundamental space of preferences as the space $\mathbf{P}_{v, d s c}$ of preferences over $V$ that satisfy A1-A8. According to Proposition $2, \mathbf{P}_{v, d s c}$ can be seen as a subspace of $\mathbf{U}_{v}$ and, hence, of $C^{2}(V)$. Our main result is that $\mathbf{P}_{v, d s c}$ is topologically complete, as desired, and that it is contractible.

Theorem $1 \mathbf{P}_{v, d s c}$ is a contractible Polish space.

Proof. (Polish) Letting $\mathbf{U}_{v, d s c} \equiv P^{-1}\left(\mathbf{P}_{v, d s c}\right)$, we get by Proposition 2 that $\mathbf{U}_{v, d s c}$ and $\mathbf{P}_{v, d s c}$ are homeomorphic under the natural map $P$, so we can indifferently work with preferences in $\mathbf{P}_{v, d s c}$ or their normalized representations in $\mathbf{U}_{v, d s c}$. We first prove that $\mathbf{U}_{v, d s c}$ is a Polish space. Let $\left(t_{n}\right)_{n \in \mathbb{N}}$ be a sequence in $\mathbb{R}$ decreasing to $\underline{t}\left(0_{\ell}\right)$, and let $\left(K_{n}\right)_{n \in \mathbb{N}}$ be an increasing sequence of compact convex sets such that $\bigcup_{n \in \mathbb{N}} K_{n}=V$. Then $\mathbf{U}_{v, d s c}$ is the intersection of the following countable families of open sets:

$$
\begin{aligned}
& \left\{u \in C^{2}(V): \frac{\partial u}{\partial t}(q, t)>0 \text { for all }(q, t) \in K_{n}\right\}, \\
& \left\{u \in C^{2}(V): \text { there exists } \varepsilon>0 \text { such that } u(q, t)<u\left(0_{\ell}, t_{n}\right)\right. \\
& \left.\quad \text { if }(q, t) \in K_{n} \text { and } \inf _{\left(q^{\prime}, t^{\prime}\right) \in \mathbb{R}^{\ell+1} \backslash V}\left\|\left(q^{\prime}, t^{\prime}\right)-(q, t)\right\| \leq \varepsilon\right\}, \\
& \left\{u \in C^{2}(V):\left|\min _{\left(0_{\ell}, t\right) \in K_{n}} u\left(0_{\ell}, t\right)-\min _{(q, t) \in K_{n}} u(q, t)\right| \vee\left|\max _{\left(0_{\ell}, t\right) \in K_{n}} u\left(0_{\ell}, t\right)-\max _{(q, t) \in K_{n}} u(q, t)\right|<\frac{1}{n}\right\}, \\
& \left\{u \in C^{2}(V): \max _{\left(0_{\ell}, t\right) \in K_{n}}\left|u\left(0_{\ell}, t\right)-t\right|<\frac{1}{n}\right\},
\end{aligned}
$$




$$
\begin{gathered}
\left\{u \in C^{2}(V): \text { there exists } \xi: u(V) \rightarrow \mathbb{R} \text { such that } \partial \xi>0 \text { over } u(V)\right. \\
\text { and } \left.\partial^{2}(\xi \circ u) \text { is negative definite over } K_{n}\right\} .
\end{gathered}
$$

The first family deals with the monotonicity of preferences with respect to transfers (A2), the second family with the boundary behavior of preferences (A4), the third and fourth families with the normalization (A6), and the fifth family with the differential strict convexity of preferences $(\mathrm{A} 7-\mathrm{A} 8)$, bearing in mind that differentiably strictly convex preferences can be represented over any compact convex subset $K$ of $V$ by a $C^{2}$ utility function $u$ with no critical point such that $\partial^{2} u$ is negative definite over $K$ (Mas-Colell (1985, Proposition 2.6.4)). Hence $\mathbf{U}_{v, d s c}$ is a $G_{\delta}$ in the Polish space $C^{2}(V)$ and thus, by Alexandrov's lemma (Mas-Colell (1985, Chapter 1, A.3.4)), a Polish space itself in the relative topology.

(Contractible) To prove that $\mathbf{U}_{v, d s c}$ is contractible, we show that the identity function over $\mathbf{U}_{v, d s c}$ is homotopic to a constant function; that is, there exists a continuous function $h_{\bar{u}}: \mathbf{U}_{v, d s c} \times[0,1] \rightarrow \mathbf{U}_{v, d s c}$ such that, for some $\bar{u} \in \mathbf{U}_{v, d s c}$, we have, for each $u \in \mathbf{U}_{v, d s c}$, $h_{\bar{u}}(u, 0)=u$ and $h_{\bar{u}}(u, 1)=\bar{u}$. Thus pick an arbitrary $\bar{u} \in \mathbf{U}_{v, d s c}$ and, to each $(u, \xi) \in$ $\mathbf{U}_{v, d s c} \times[0,1]$, associate a utility function $u_{\xi}$ as follows. First, let $u_{0} \equiv u$ and $u_{1} \equiv \bar{u}$. Then, for all $\xi \in(0,1)$ and $(q, t) \in V$, consider the equation in $\mu$ :

$$
\bar{u}(q, \mu)=u\left(q, \frac{t-\xi \mu}{1-\xi}\right) .
$$

We claim that (1) has a unique solution in the admissible range for $\mu$,

$$
\underline{t}(q)<\mu<\underline{t}(q)+\frac{1}{\xi}[t-\underline{t}(q)]
$$

with $-\infty+\infty / \xi=\infty$ by convention. Indeed, the left-hand side of (1) is strictly increasing in $\mu$, whereas the right-hand side of (1) is strictly decreasing in $\mu$. Moreover, as both $u$ and $\bar{u}$ belong to $\mathbf{U}_{v, d s c}$, we have $\inf \operatorname{range} u(q, \cdot)=\inf$ range $\bar{u}(q, \cdot)=\underline{t}\left(0_{\ell}\right)$. Therefore,

$$
\lim _{\mu \downarrow \underline{t}(q)} \bar{u}(q, \mu)=\underline{t}\left(0_{\ell}\right)<\lim _{\mu \downarrow \underline{t}(q)} u\left(q, \frac{t-\xi \mu}{1-\xi}\right),
$$

whereas

$$
\lim _{\mu \uparrow \underline{t}(q)+\frac{1}{\xi}[t-\underline{t}(q)]} \bar{u}(q, \mu)>\underline{t}\left(0_{\ell}\right)=\lim _{\mu \uparrow \underline{t}(q)+\frac{1}{\xi}[t-\underline{t}(q)]} u\left(q, \frac{t-\xi \mu}{1-\xi}\right),
$$

so that there exists a unique solution $\mu_{\xi}(q, t)$ to $(1)$, as claimed. We can then let $u_{\xi}(q, t) \equiv$ $\bar{u}\left(q, \mu_{\xi}(q, t)\right)$. Geometrically, what this transformation does is that, to each $t^{\prime}$ such that $\left(0_{\ell}, t^{\prime}\right) \in V$, it assigns an indifference set $u_{\xi}^{-1}\left(\left\{t^{\prime}\right\}\right)$ that is the vertical convex combination of 
the indifference sets $\bar{u}^{-1}\left(\left\{t^{\prime}\right\}\right)$ and $u^{-1}\left(\left\{t^{\prime}\right\}\right)$ with weights $\xi$ and $1-\xi$, respectively, bearing in mind that, by normalization, $\bar{u}\left(0_{\ell}, t^{\prime}\right)=u\left(0_{\ell}, t^{\prime}\right)=t^{\prime}$. Figure 3 below illustrates this transformation.

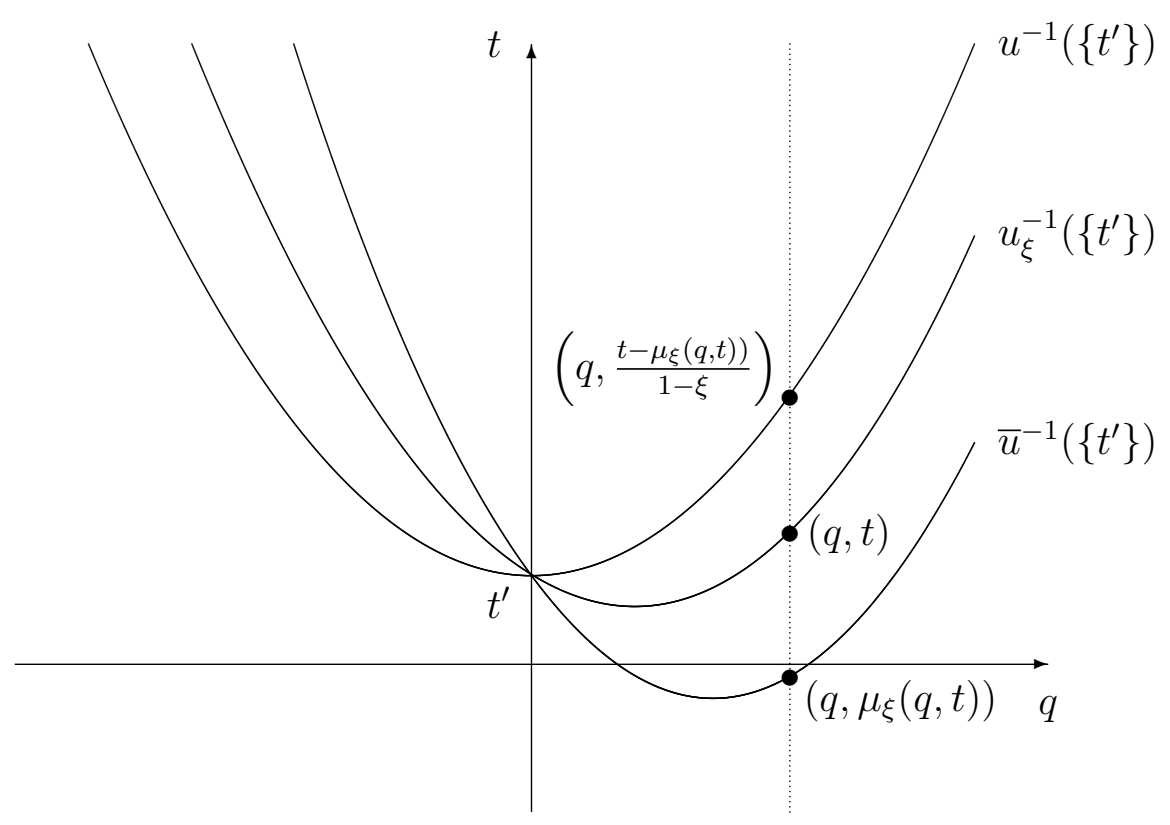

Figure 3 The homotopy $h_{\bar{u}}$.

To complete the proof, we show that the mapping $(u, \xi) \mapsto u_{\xi}$ yields the desired homotopy. The proof consists of two steps.

Step 1 We first verify that $u_{\xi} \in \mathbf{U}_{v, d s c}$ for all $\xi \in[0,1]$. This is obvious for $\xi=0,1$. Now fix some $\xi \in(0,1)$. We must prove that range $u_{\xi}(q, \cdot)=$ range $u_{\xi}\left(0_{\ell}, \cdot\right)$ for all $q \in Q$ and that $u_{\xi}\left(0_{\ell}, t\right)=t$ for all $t$ such that $\left(0_{\ell}, t\right) \in V$, that $u_{\xi}$ is $C^{2}$, with $\partial u_{\xi} / \partial t>0$ over $V$, and strictly quasiconcave, that $u_{\xi}^{-1}([v, \infty))$ is closed in $V$ for all $v \in \mathbb{R}$, and that the curvature of the indifference sets of $u_{\xi}$ nowhere vanishes.

(Normalization) By construction, we have range $u_{\xi}(q, \cdot)=\operatorname{range} u(q, \cdot)=\operatorname{range} \bar{u}(q, \cdot)=$ $\left(\underline{t}\left(0_{\ell}\right), \infty\right)$ for all $q \in Q$, and, moreover, $\left(0_{\ell}, t\right) \in u_{\xi}^{-1}(\{t\})$ for all $t>\underline{t}\left(0_{\ell}\right)$. Hence $u_{\xi}$ is normalized, as desired.

(Regularity) By (1), for each $(q, t) \in V, \mu_{\xi}(q, t)$ is the unique solution to $f(q, t, \mu)=0$, where $f(q, t, \mu) \equiv \bar{u}(q, \mu)-u(q,(t-\xi \mu) /(1-\xi))$. That $\mu_{\xi}$ is $C^{2}$ follows from the implicit function theorem along with the fact that $\partial f / \partial \mu>0$ as $\partial \bar{u} / \partial t>0$ and $\partial u / \partial t>0$ over $V$. That $u_{\xi}$ is $C^{2}$ then follows from the identity $u_{\xi}(q, t) \equiv \bar{u}\left(q, \mu_{\xi}(q, t)\right)$. 
(Monotonicity in transfers) Differentiating the identity $f\left(q, t, \mu_{\xi}(q, t)\right)=0$ and using the fact that $\partial \bar{u} / \partial t>0$ and $\partial u / \partial t>0$ over $V$ yields, for each $(q, t) \in V$,

$$
\frac{\partial \mu_{\xi}}{\partial t}(q, t)=\frac{\frac{\partial u}{\partial t}\left(q, \frac{t-\xi \mu_{\xi}(q, t)}{1-\xi}\right)}{\xi \frac{\partial u}{\partial t}\left(q, \frac{t-\xi \mu_{\xi}(q, t)}{1-\xi}\right)+(1-\xi) \frac{\partial \bar{u}}{\partial t}\left(q, \mu_{\xi}(q, t)\right)}>0 .
$$

That $\partial u_{\xi} / \partial t>0$ over $V$ then follows from from A2 and the identity $u_{\xi}(q, t) \equiv \bar{u}\left(q, \mu_{\xi}(q, t)\right)$.

(Strict quasiconcavity) By construction, for each $t^{\prime}$ such that $\left(0_{\ell}, t^{\prime}\right) \in V$, the indifference sets $\bar{u}^{-1}\left(\left\{t^{\prime}\right\}\right), u^{-1}\left(\left\{t^{\prime}\right\}\right)$, and $u_{\xi}^{-1}\left(\left\{t^{\prime}\right\}\right)$ can be parameterized as $t=\bar{\tau}\left(q, t^{\prime}\right), t=\tau\left(q, t^{\prime}\right)$, and $t=\tau_{\xi}\left(q, t^{\prime}\right)=\xi \bar{\tau}\left(q, t^{\prime}\right)+(1-\xi) \tau\left(q, t^{\prime}\right)$, respectively. Because $\bar{u}$ and $u$ are strictly quasiconcave, the mappings $q \mapsto \bar{\tau}\left(q, t^{\prime}\right)$ and $q \mapsto \tau\left(q, t^{\prime}\right)$ are strictly convex, and so is the mapping $q \mapsto \tau_{\xi}\left(q, t^{\prime}\right)$ by convex combination. That $u_{\xi}$ is strictly quasiconcave then follows from this observation along with the monotonicity of $u_{\xi}$ in transfers.

(Boundary behavior) Fix some $v \in$ range $u_{\xi}=$ range $u=\operatorname{range} \bar{u}$ and let $\left(\left(q_{n}, t_{n}\right)\right)_{n \in \mathbb{N}}$ be a sequence in $u_{\xi}^{-1}([v, \infty))$ that converges to $(q, t) \in \mathbb{R}^{\ell+1}$. We must prove that $(q, t) \in$ $u_{\xi}^{-1}([v, \infty))$. Because $u_{\xi}$ is continuous over $V$, we only need to check that $(q, t)$ does not belong to the boundary of $V$ in $\mathbb{R}^{\ell+1}$. Recall first that, by construction, $\left(q_{n}, t_{n}\right)$ is for each $n \in \mathbb{N}$ a convex combination with weights $\xi$ and $1-\xi$ of $\left(q_{n}, \mu_{\xi}\left(q_{n}, t_{n}\right)\right) \in \bar{u}^{-1}([v, \infty))$ and $\left(q_{n},\left[t_{n}-\xi \mu_{\xi}\left(q_{n}, t_{n}\right)\right] /(1-\xi)\right) \in u^{-1}([v, \infty))$. We show that an implication of this is that the sequences $\left(\left(q_{n}, \mu_{\xi}\left(q_{n}, t_{n}\right)\right)\right)_{n \in \mathbb{N}}$ and $\left(\left(q_{n},\left[t_{n}-\xi \mu_{\xi}\left(q_{n}, t_{n}\right)\right] /(1-\xi)\right)\right)_{n \in \mathbb{N}}$ remain bounded. Indeed, if they are not, then, as the sequence $\left(\left(q_{n}, t_{n}\right)\right)_{n \in \mathbb{N}}$ is bounded, we can extract two divergent subsequences $\left(\mu_{\xi}\left(q_{n_{k}}, t_{n_{k}}\right)\right)_{k \in \mathbb{N}}$ and $\left(\left[t_{n_{k}}-\xi \mu_{\xi}\left(q_{n_{k}}, t_{n_{k}}\right)\right] /(1-\xi)\right)_{k \in \mathbb{N}}$ of transfers with opposite signs; suppose with no loss of generality that $\lim _{k \rightarrow \infty} \mu_{\xi}\left(q_{n_{k}}, t_{n_{k}}\right)=-\infty$. Now, fix some $\left(q^{\prime}, t^{\prime}\right)$ such that $\bar{u}\left(q^{\prime}, t^{\prime}\right)=v$. Then, because $\bar{u}$ is quasiconcave,

$$
\left[1+\frac{1}{\mu_{\xi}\left(q_{n_{k}}, t_{n_{k}}\right)}\right]\left(q^{\prime}, t^{\prime}\right)-\frac{1}{\mu_{\xi}\left(q_{n_{k}}, t_{n_{k}}\right)}\left(q_{n_{k}}, \mu_{\xi}\left(q_{n_{k}}, t_{n_{k}}\right)\right) \in \bar{u}^{-1}([v, \infty))
$$

for all $k$ such that $\mu_{\xi}\left(q_{n_{k}}, t_{n_{k}}\right)<-1$. Letting $k$ go to $\infty$, we get by continuity of $\bar{u}$ that $\bar{u}\left(q^{\prime}, t^{\prime}-1\right) \geq v=\bar{u}\left(q^{\prime}, t^{\prime}\right)$, which contradicts the fact that $\bar{u}\left(q^{\prime}, \cdot\right)$ is strictly increasing. Thus both $\left(\left(q_{n}, \mu_{\xi}\left(q_{n}, t_{n}\right)\right)\right)_{n \in \mathbb{N}}$ and $\left(\left(q_{n},\left[t_{n}-\xi \mu_{\xi}\left(q_{n}, t_{n}\right)\right] /(1-\xi)\right)\right)_{n \in \mathbb{N}}$ remain bounded, as claimed. Extracting subsequences if necessary, let us denote by $\left(q, t_{\bar{u}}\right)$ and $\left(q, t_{u}\right)$ their respective limits; suppose with no loss of generality that $t_{\bar{u}} \leq t \leq t_{u}$. As $\bar{u}^{-1}([v, \infty))$ is closed and comprehensive, and as $\left(q_{n}, \mu_{\xi}\left(q_{n}, t_{n}\right)\right) \in \bar{u}^{-1}([v, \infty))$ for all $n \in \mathbb{N}$, we have $\left(q, t_{\bar{u}}\right) \in \bar{u}^{-1}([v, \infty))$ and, hence, $(q, t) \in \bar{u}^{-1}([v, \infty))$. That $(q, t)$ cannot belong to the common boundary of $\mathbb{R}^{\ell+1} \backslash V$ and $V$ in $\mathbb{R}^{\ell+1}$ then follows from the fact that the disjoint 
closed sets $\bar{u}^{-1}([v, \infty))$ and $\mathbb{R}^{\ell+1} \backslash V$ can be separated by open sets in $\mathbb{R}^{\ell+1}$.

(Curvature) Given the parametrization $t=\tau_{\xi}\left(q, t^{\prime}\right)=\xi \bar{\tau}\left(q, t^{\prime}\right)+(1-\xi) \tau\left(q, t^{\prime}\right)$ of the $t^{\prime}$-indifference set $u_{\xi}^{-1}\left(\left\{t^{\prime}\right\}\right)$ of $u_{\xi}$, the Hessian $\partial^{2} \tau_{\xi}\left(q, t^{\prime}\right)=\xi \partial^{2} \bar{\tau}\left(q, t^{\prime}\right)+(1-\xi) \partial^{2} \tau\left(q, t^{\prime}\right)$ is positive definite, because so must be the Hessians $\partial^{2} \bar{\tau}\left(q, t^{\prime}\right)$ and $\partial^{2} \tau\left(q, t^{\prime}\right)$ for the curvatures of the $t^{\prime}$-indifference sets $\bar{u}^{-1}\left(\left\{t^{\prime}\right\}\right)$ of $\bar{u}$ and $u^{-1}\left(\left\{t^{\prime}\right\}\right)$ of $u$ to nowhere vanish (Mas-Colell (1985, Chapter 1, H.3)).

Step 2 There remains to check that the mapping $h_{\bar{u}}: \mathbf{U}_{v, d s c} \times[0,1] \rightarrow \mathbf{U}_{v, d s c}:(u, \xi) \mapsto u_{\xi}$ is continuous. Let $\left(\left(u_{n}, \xi_{n}\right)\right)_{n \in \mathbb{N}}$ be a sequence in $\mathbf{U}_{v, d s c} \times[0,1]$ converging to $(u, \xi)$. To avoid trivial cases, let us assume that for each $n \in \mathbb{N}$, there exists $m \geq n$ such that $\xi_{m} \neq 0,1$. We must prove that the sequences $\left(h_{\bar{u}}\left(u_{n}, \xi_{n}\right)\right)_{n \in \mathbb{N}},\left(\partial h_{\bar{u}}\left(u_{n}, \xi_{n}\right)\right)_{n \in \mathbb{N}}$, and $\left(\partial^{2} h_{\bar{u}}\left(u_{n}, \xi_{n}\right)\right)_{n \in \mathbb{N}}$ converge to $h_{\bar{u}}(u, \xi), \partial h_{\bar{u}}(u, \xi)$, and $\partial^{2} h_{\bar{u}}(u, \xi)$, respectively, uniformly over any compact subset $K$ of $V$. The proof consists of three substeps.

Step 2.1 For each $n \in \mathbb{N}$ such that $\xi_{n} \in(0,1)$, and for each $(q, t) \in K$, define $\mu_{n, \xi_{n}}(q, t)$ as the unique solution to

$$
\bar{u}(q, \mu)=u_{n}\left(q, \frac{t-\xi_{n} \mu}{1-\xi_{n}}\right) .
$$

We first claim that the sequences $\left(\mu_{n, \xi_{n}}(q, t)\right)_{n \in \mathbb{N}}$ and $\left(\left[t-\xi_{n} \mu_{n, \xi_{n}}(q, t)\right] /\left(1-\xi_{n}\right)\right)_{n \in \mathbb{N}}$ are bounded, uniformly in $(q, t) \in K$. By way of contradiction, let us assume, for instance, that there exists a divergent sequence $\left(\mu_{n_{k}, \xi_{n_{k}}}\left(q_{k}, t_{k}\right)\right)_{k \in \mathbb{N}}$ with $\left(q_{k}, t_{k}\right) \in K$ for all $k \in \mathbb{N}$; suppose with no loss of generality that the sequence $\left(\left(q_{k}, t_{k}\right)\right)_{k \in \mathbb{N}}$ converges to some $(q, t) \in K$ and that $\lim _{k \rightarrow \infty} \mu_{n_{k}, \xi_{n_{k}}}\left(q_{k}, t_{k}\right)=-\infty$. (The other cases can be handled in a similar way.) Then, as the sequence $\left(q_{k}\right)_{k \in \mathbb{N}}$ converges to $q$, we have $\underline{t}(q)=-\infty$ and, hence, $\underline{t}\left(0_{\ell}\right)=-\infty$, which in turn implies that range $\bar{u}(q, \cdot)=$ range $\bar{u}\left(0_{\ell}, \cdot\right)=\mathbb{R}$ by normalization. Now, for each $\left(t^{\prime}, \varepsilon\right) \in \mathbb{R} \times \mathbb{R}_{++}$, we have $\mu_{n_{k}, \xi_{n_{k}}}\left(q_{k}, t_{k}\right) \leq t^{\prime}$ and $\bar{u}\left(q_{k}, \mu_{n_{k}, \xi_{n_{k}}}\left(q_{k}, t_{k}\right)\right) \leq \bar{u}\left(q, t^{\prime}\right)+\varepsilon$ for $k$ large enough. Because $t^{\prime}$ can take any value in $\mathbb{R}$ and range $\bar{u}(q, \cdot)=\mathbb{R}$, we obtain

$$
\lim _{k \rightarrow \infty} \bar{u}\left(q_{k}, \mu_{n_{k}, \xi_{n_{k}}}\left(q_{k}, t_{k}\right)\right)=-\infty .
$$

On the other hand, as the sequence $\left(t_{k}\right)_{k \in \mathbb{N}}$ converges, $\lim _{k \rightarrow \infty} \mu_{n_{k}, \xi_{n_{k}}}\left(q_{k}, t_{k}\right)=-\infty$ implies that $\mu_{n_{k}, \xi_{n_{k}}}\left(q_{k}, t_{k}\right) \leq t_{k}$ and, hence, $\left[t_{k}-\xi_{n_{k}} \mu_{n_{k}, \xi_{n_{k}}}\left(q_{k}, t_{k}\right)\right] /\left(1-\xi_{n_{k}}\right) \geq t_{k}$ for $k$ large enough. Thus we obtain

$$
\liminf _{k \rightarrow \infty} u_{n_{k}}\left(q_{k}, \frac{t_{k}-\xi_{n_{k}} \mu_{n_{k}, \xi_{n_{k}}}\left(q_{k}, t_{k}\right)}{1-\xi_{n_{k}}}\right) \geq \lim _{k \rightarrow \infty} u_{n_{k}}\left(q_{k}, t_{k}\right)=u(q, t),
$$

where the equality follows from the fact that the sequence $\left(u_{n}\right)_{n \in \mathbb{N}}$ converges uniformly to $u$ over $K$ and that the sequence $\left(\left(q_{k}, t_{k}\right)\right)_{k \in \mathbb{N}}$ converges to $(q, t)$ in $K$. But then, in light 
of (3)-(4), (2) cannot hold for $(n, q, t, \mu)=\left(n_{k}, q_{k}, t_{k}, \mu_{n_{k}, \xi_{n_{k}}}\left(q_{k}, t_{k}\right)\right)$ for $k$ large enough, a contradiction. The claim follows.

Step 2.2 We now establish that the sequence $\left(\mu_{n, \xi_{n}}\right)_{n \in \mathbb{N}}$ converges uniformly to $\mu_{\xi}$ over $K$ when the sequence $\left(\xi_{n}\right)_{n \in \mathbb{N}}$ converges to $\xi \in(0,1)$, so that $\xi_{n} \neq 0,1$ for $n \in \mathbb{N}$ large enough. For any such $n$, and for each $(q, t) \in K$, we have, by (2),

$$
\bar{u}\left(q, \mu_{n, \xi_{n}}(q, t)\right)-u\left(q, \frac{t-\xi \mu_{n, \xi_{n}}(q, t)}{1-\xi}\right)=\Delta_{1, n}(q, t)+\Delta_{2, n}(q, t),
$$

where

$$
\Delta_{1, n}(q, t) \equiv u_{n}\left(q, \frac{t-\xi_{n} \mu_{n, \xi_{n}}(q, t)}{1-\xi_{n}}\right)-u\left(q, \frac{t-\xi_{n} \mu_{n, \xi_{n}}(q, t)}{1-\xi_{n}}\right)
$$

and

$$
\Delta_{2, n}(q, t) \equiv u\left(q, \frac{t-\xi_{n} \mu_{n, \xi_{n}}(q, t)}{1-\xi_{n}}\right)-u\left(q, \frac{t-\xi \mu_{n, \xi_{n}}(q, t)}{1-\xi}\right) .
$$

According to Step 2.1, there exists some compact subset $K^{\prime}$ of $V$ such that

$$
\max _{(q, t) \in K}\left|\Delta_{1, n}(q, t)\right| \leq\left\|u_{n}-u\right\|_{K^{\prime}}
$$

and

$$
\max _{(q, t) \in K}\left|\Delta_{2, n}(q, t)\right| \leq\left\|\frac{\partial u}{\partial t}\right\|_{K^{\prime}} \sup _{(q, t) \in K} \frac{\left|\left(\xi_{n}-\xi\right)\left[t-\mu_{n, \xi_{n}}(q, t)\right]\right|}{(1-\xi)\left(1-\xi_{n}\right)}
$$

Taking limits yields, by (5),

$$
\begin{aligned}
\lim _{n \rightarrow \infty} \bar{u}\left(q, \mu_{n, \xi_{n}}(q, t)\right)-u\left(q, \frac{t-\xi \mu_{n, \xi_{n}}(q, t)}{1-\xi}\right) & =0 \\
& =\bar{u}\left(q, \mu_{\xi}(q, t) t\right)-u\left(q, \frac{t-\xi \mu_{\xi}(q, t)}{1-\xi}\right)
\end{aligned}
$$

uniformly in $(q, t) \in K$. According to Step 2.1, this implies that

$$
\lim _{n \rightarrow \infty}\left[\min _{\left(q^{\prime}, t^{\prime}\right) \in K^{\prime}} \frac{\partial \bar{u}}{\partial t}\left(q^{\prime}, t^{\prime}\right)+\frac{\xi}{1-\xi} \min _{\left(q^{\prime}, t^{\prime}\right) \in K^{\prime}} \frac{\partial u}{\partial t}\left(q^{\prime}, t^{\prime}\right)\right]\left|\mu_{n, \xi_{n}}(q, t)-\mu_{\xi}(q, t)\right|=0,
$$

uniformly in $(q, t) \in K$, from which the claim follows as both $\partial \bar{u} / \partial t$ and $\partial u / \partial t$ are positive and bounded away from 0 over any compact subset of $V$.

Step 2.3 We can now establish the desired convergence results, first for the functions $\left(h_{\bar{u}}\left(u_{n}, \xi_{n}\right)\right)_{n \in \mathbb{N}}$, and then for their first- and second-order derivatives $\left(\partial h_{\bar{u}}\left(u_{n}, \xi_{n}\right)\right)_{n \in \mathbb{N}}$ and $\left(\partial^{2} h_{\bar{u}}\left(u_{n}, \xi_{n}\right)\right)_{n \in \mathbb{N}}$. 
(Functions) Suppose first that the sequence $\left(\xi_{n}\right)_{n \in \mathbb{N}}$ converges to $\xi \in(0,1)$. For all $(q, t) \in$ $K$ and $n \in \mathbb{N}$, we have $h_{\bar{u}}\left(u_{n}, \xi_{n}\right)(q, t)=\bar{u}\left(q, \mu_{n, \xi_{n}}(q, t)\right)$ and $h_{\bar{u}}(u, \xi)(q, t)=\bar{u}\left(q, \mu_{\xi}(q, t)\right)$. Hence, by Step 2.1,

$$
\left\|h_{\bar{u}}\left(u_{n}, \xi_{n}\right)-h_{\bar{u}}(u, \xi)\right\|_{K} \leq\left\|\frac{\partial \bar{u}}{\partial t}\right\|_{K^{\prime}}\left\|\mu_{n, \xi_{n}}-\mu_{\xi}\right\|_{K},
$$

which converges to 0 according to Step 2.2. Suppose next that the sequence $\left(\xi_{n}\right)_{n \in \mathbb{N}}$ converges to 0 . We can focus on the terms of the sequence $\left(h_{\bar{u}}\left(u_{n}, \xi_{n}\right)\right)_{n \in \mathbb{N}}$ such that $\xi_{n} \neq 0$, for the other terms are equal to $h_{\bar{u}}\left(u_{n}, 0\right)=u_{n}$, and the sequence $\left(u_{n}\right)_{n \in \mathbb{N}}$ converges uniformly over $K$ to $u=h_{\bar{u}}(u, 0)$. Then (5) holds for $\xi=0$, and reasoning as in Step 2.2 yields that

$$
\left\|h_{\bar{u}}\left(u_{n}, \xi_{n}\right)-u\right\|_{K}=\left\|h_{\bar{u}}\left(u_{n}, \xi_{n}\right)-h_{\bar{u}}(u, 0)\right\|_{K}
$$

converges to 0 . Suppose finally that the sequence $\left(\xi_{n}\right)_{n \in \mathbb{N}}$ converges to 1 . We can focus on the terms of the sequence $\left(h_{\bar{u}}\left(u_{n}, \xi_{n}\right)\right)_{n \in \mathbb{N}}$ such that $\xi_{n} \neq 1$, for the other terms are equal to $h_{\bar{u}}\left(u_{n}, 1\right)=\bar{u}$. Then, letting $\nu_{n, \xi_{n}}(q, t) \equiv\left[t-\xi_{n} \mu_{n, \xi_{n}}(q, t)\right] /\left(1-\xi_{n}\right)$ for all $(q, t) \in K$, we have $h_{\bar{u}}\left(u_{n}, \xi_{n}\right)=\bar{u}\left(q,\left[t-\left(1-\xi_{n}\right) \nu_{n, \xi_{n}}(q, t)\right] / \xi_{n}\right)$. Bearing in mind that, as established in Step 2.1 , the sequence $\left(\nu_{n, \xi_{n}}(q, t)\right)_{n \in \mathbb{N}}$ is bounded, uniformly in $(q, t) \in K$, we obtain that

$$
\left\|h_{\bar{u}}\left(u_{n}, \xi_{n}\right)-\bar{u}\right\|_{K}=\left\|h_{\bar{u}}\left(u_{n}, \xi_{n}\right)-h_{\bar{u}}(u, 1)\right\|_{K}
$$

converges to 0 . Therefore, in any case, the sequence $\left(h_{\bar{u}}\left(u_{n}, \xi_{n}\right)\right)_{n \in \mathbb{N}}$ converges to $h_{\bar{u}}(u, \xi)$ uniformly over $K$, as desired.

(Derivatives) We focus on the first-order derivatives $\left(\partial h_{\bar{u}}\left(u_{n}, \xi_{n}\right)\right)_{n \in \mathbb{N}}$. (The proof for the second-order derivatives $\left(\partial^{2} h_{\bar{u}}\left(u_{n}, \xi_{n}\right)\right)_{n \in \mathbb{N}}$ is similar and is, therefore, omitted.) Suppose first that the sequence $\left(\xi_{n}\right)_{n \in \mathbb{N}}$ converges to $\xi \in(0,1)$, so that $\xi_{n} \neq 0,1$ for $n \in \mathbb{N}$ large enough. For any such $n$, and for each $(q, t) \in K$, we have, by the implicit function theorem,

$$
\left(\begin{array}{c}
\vdots \\
\frac{\partial \mu_{n, \xi_{n}}}{\partial q_{l}}(q, t) \\
\vdots \\
\frac{\partial \mu_{n, \xi_{n}}}{\partial t}(q, t)
\end{array}\right)=\left(\begin{array}{c}
\vdots \\
\frac{\left(1-\xi_{n}\right)\left[\frac{\partial u_{n}}{\partial q_{l}}\left(q, \frac{t-\xi_{n} \mu_{n, \xi_{n}}(q, t)}{1-\xi_{n}}\right)-\frac{\partial \bar{u}}{\partial q_{l}}\left(q, \mu_{n, \xi_{n}}(q, t)\right)\right]}{\xi_{n} \frac{\partial u_{n}}{\partial t}\left(q, \frac{t-\xi_{n} \mu_{n, \xi_{n}}(q, t)}{1-\xi_{n}}\right)+\left(1-\xi_{n}\right) \frac{\partial \bar{u}}{\partial t}\left(q, \mu_{n, \xi_{n}}(q, t)\right)} \\
\vdots \\
\frac{\partial u_{n}}{\partial t}\left(q, \frac{t-\xi_{n} \mu_{n, \xi_{n}}(q, t)}{1-\xi_{n}}\right) \\
\frac{\xi_{n} \frac{\partial u_{n}}{\partial t}\left(q, \frac{t-\xi_{n} \mu_{n, \xi_{n}}(q, t)}{1-\xi_{n}}\right)+\left(1-\xi_{n}\right) \frac{\partial \bar{u}}{\partial t}\left(q, \mu_{n, \xi_{n}}(q, t)\right)}{2}
\end{array}\right)
$$

Because, as established in Step 2.2, the sequence $\left(\mu_{n, \xi_{n}}\right)_{n \in \mathbb{N}}$ converges uniformly to $\mu_{\xi}$ over $K$, and the sequences $\left(\partial u_{n} / \partial q_{l}\right)_{n \in \mathbb{N}}$ and $\left(\partial u_{n} / \partial t\right)_{n \in \mathbb{N}}$ converge uniformly to $\partial u / \partial q_{l}$ and $\partial u / \partial t$ 
over compact subsets of $V$, this converges to

$$
\left(\begin{array}{c}
\frac{(1-\xi)\left[\frac{\partial u}{\partial q_{l}}\left(q, \frac{t-\xi \mu_{\xi}(q, t)}{1-\xi}\right)-\frac{\partial \bar{u}}{\partial q_{l}}\left(q, \mu_{\xi}(q, t)\right)\right]}{\xi \frac{\partial u}{\partial t}\left(q, \frac{t-\xi \mu_{\xi}(q, t)}{1-\xi}\right)+(1-\xi) \frac{\partial \bar{u}}{\partial t}\left(q, \mu_{\xi}(q, t)\right)} \\
\vdots \\
\frac{\frac{\partial u}{\partial t}\left(q, \frac{t-\xi \mu_{\xi}(q, t)}{1-\xi}\right)}{\xi \frac{\partial u}{\partial t}\left(q, \frac{t-\xi \mu_{\xi}(q, t)}{1-\xi}\right)+(1-\xi) \frac{\partial \bar{u}}{\partial t}\left(q, \mu_{\xi}(q, t)\right)}
\end{array}\right)=\left(\begin{array}{c}
\vdots \\
\frac{\partial \mu_{\xi}}{\partial q_{l}}(q, t) \\
\vdots \\
\frac{\partial \mu_{\xi}}{\partial t}(q, t)
\end{array}\right)
$$

uniformly in $(q, t) \in K$. As a result,

$$
\partial h_{\bar{u}}\left(u_{n}, \xi_{n}\right)(q, t)=\left(\begin{array}{c}
\vdots \\
\frac{\partial \bar{u}}{\partial q_{l}}\left(q, \mu_{n, \xi_{n}}(q, t)\right)+\frac{\partial \bar{u}}{\partial t}\left(q, \mu_{n, \xi_{n}}(q, t)\right) \frac{\partial \mu_{n, \xi_{n}}}{\partial q_{l}}(q, t) \\
\vdots \\
\frac{\partial \bar{u}}{\partial t}\left(q, \mu_{n, \xi_{n}}(q, t)\right) \frac{\partial \mu_{n, \xi_{n}}}{\partial t}(q, t)
\end{array}\right)
$$

converges to

$$
\left(\begin{array}{c}
\frac{\partial \bar{u}}{\partial q_{l}}\left(q, \mu_{\xi}(q, t)\right)+\frac{\partial \bar{u}}{\partial t}\left(q, \mu_{\xi}(q, t)\right) \frac{\partial \mu_{\xi}}{\partial q_{l}}(q, t) \\
\vdots \\
\frac{\partial \bar{u}}{\partial t}\left(q, \mu_{\xi}(q, t)\right) \frac{\partial \mu_{\xi}}{\partial t}(q, t)
\end{array}\right)=\partial h_{\bar{u}}(u, \xi)(q, t)
$$

uniformly in $(q, t) \in K$. Suppose finally that the sequence $\left(\xi_{n}\right)_{n \in \mathbb{N}}$ converges to 0 . (The proof for the case where the sequence $\left(\xi_{n}\right)_{n \in \mathbb{N}}$ converges to 1 is similar and is, therefore, omitted.) We can focus on the terms of the sequence $\left(\partial h_{\bar{u}}\left(u_{n}, \xi_{n}\right)\right)_{n \in \mathbb{N}}$ such that $\xi_{n} \neq 0$, for the other terms are equal to $\partial h_{\bar{u}}\left(u_{n}, 0\right)=\partial u_{n}$, and the sequence $\left(\partial u_{n}\right)_{n \in \mathbb{N}}$ converges uniformly over $K$ to $\partial u=\partial h_{\bar{u}}(u, 0)$. For any such $n$, and for each $(q, t) \in K$, we have

$$
\partial h_{\bar{u}}\left(u_{n}, \xi_{n}\right)(q, t)=\partial \bar{u}\left(q, \mu_{n, \xi_{n}}(q, t)\right)=\partial u_{n}\left(q, \frac{t-\xi_{n} \mu_{n, \xi_{n}}(q, t)}{1-\xi_{n}}\right),
$$

Because, as established in Step 2.1, the sequence $\left(\mu_{n, \xi_{n}}(q, t)\right)_{n \in \mathbb{N}}$ is bounded, uniformly in $(q, t) \in K$, and the sequence $\left(\partial u_{n}\right)_{n \in \mathbb{N}}$ converges uniformly to $\partial u$ over compact subsets of $V$, this converges to $\partial u(q, t)$, uniformly in $(q, t) \in K$. Hence the result.

Observe that the compensation principle expressed by A6 plays a key role in Step 2 of the proof of Theorem 1 by ensuring that the vertical convex combination of any two indifference curves for $u$ and $\bar{u}$ going through the same point of the vertical axis is well defined, which 
in turn allows us to construct the desired contraction $h_{\bar{u}}$ in a straightforward way. It follows from the proof of Theorem 1 that the space $\mathbf{P}_{d s c}$ of preferences that satisfy $\mathrm{A} 1-\mathrm{A} 5$ and A7-A8, but not necessarily A6, is topologically complete. However, it is an open question whether it is contractible.

\section{Collective Choice}

We now draw the implications of our analysis for the aggregation of individual preference relations in $\mathbf{P}_{v, d s c}$. The social-choice framework we have in mind is as follows. Consider a group of $I$ individuals $i=1, \ldots, I$, which may be thought of as a household, a family, a firm, a union, or a club. Each individual in the group has preferences about the consumption of private and collective goods within the group. An individual may in particular care for the consumption of private goods by other members of the group, as in Becker's (1981) model of altruism in the family or Chiappori's $(1988,1992)$ collective model of household labor supply. Individuals can also contribute to the supply of collective goods, such as household chores or meeting participations. It is realistic to assume that their preferences with respect to such contributions will be nonmonotone: a small contribution by an individual comes at a negligible marginal cost, while generating a substantial marginal benefit to him, both directly and perhaps indirectly through altruistic concerns; however, large contributions will typically generate substantial costs that outweighs personal benefits. ${ }^{5}$ Finally, we assume that, whereas individuals may differ as to the values they attribute to the consumption of private and collective goods within the group, as well as to their respective contributions to the supply of collective goods, there is one collectively consumed good which is always desirable from each individual's viewpoint. An example in the case of a household or a family may be an index of the quantity and quality of time spent together on vacation; in the case of a club, the practice of common-interest activities.

Formally, we will suppose that, as in the general model of Section 2, individuals have preferences defined over $\ell$ commodities representing the amounts of private and collective goods consumed within the group, as well as the privately borne contributions to the latter, and a desirable $\ell+1^{\text {th }}$ collective good. Each individual $i$ is endowed with a preference relation $\succeq_{i} \in \mathbf{P}_{v, d s c}$ over the relevant consumption set $V$. The question we ask ourselves is how to aggregate a profile of individual preferences relations in $\mathbf{P}_{v, d s c}$ into a collective preference

\footnotetext{
${ }^{5}$ Conversely, negative contributions to collective goods can, in the presence of altruistic concerns, come at a cost in the form of guilt or shame; this can be formally captured by assuming that individual upper contour sets are closed in the embedding space, as in A4.
} 
relation in $\mathbf{P}_{v, d s c}$ through a collective choice rule

$$
\Phi_{I}: \mathbf{P}_{v, d s c}^{I} \rightarrow \mathbf{P}_{v, d s c}:\left(\succeq_{1}, \ldots, \succeq_{I}\right) \mapsto \Phi_{I}\left(\succeq_{1}, \ldots, \succeq_{I}\right)
$$

To narrow down the set of admissible collective choice rules, we need to impose a number of axioms on $\Phi_{I}$. Following Chichilnisky's (1980) classical formulation of the topological socialchoice problem, we restrict ourselves to collective choice rules that satisfy the following requirements:

Anonymity For any permutation $\sigma$ of $\{1, \ldots, I\}$ and for any profile of individual preference relations $\left(\succeq_{1}, \ldots, \succeq_{I}\right) \in \mathbf{P}_{v, d s c}^{I}$,

$$
\Phi_{I}\left(\succeq_{\sigma(1)}, \ldots, \succeq_{\sigma(I)}\right)=\Phi_{I}\left(\succeq_{1}, \ldots, \succeq_{I}\right)
$$

Unanimity For any individual preference relation $\succeq \in \mathbf{P}_{v, d s c}$,

$$
\Phi_{I}(\succeq, \ldots, \succeq)=\succeq
$$

Continuity $\Phi_{I}$ is continuous.

Anonymity requires that the collective preference relation remain unchanged whenever individuals exchange their preference relations; this condition is stronger than Arrow's (1951) nondictatorship axiom. Unanimity requires that if all individuals have the same individual preference relation, then the collective preference relation must coincide with the latter; this condition is weaker than Arrow's (1951) Pareto axiom. Continuity requires that, if two profiles of individual preference relations are close enough to each other, then so must be the corresponding collective preference relations; this replaces Arrow's (1951) independence axiom as the interprofile consistency condition. ${ }^{6}$

The key question is then whether there exists a collective choice rule $\Phi_{I}$ over $\mathbf{P}_{v, d s c}^{I}$ that is continuous, anonymous, and respects unanimity. Because, according to Theorem $1, \mathbf{P}_{v, d s c}$ is contractible, it is tempting to invoke Chichilnisky and Heal (1983, Theorem 1) to infer that there indeed exists such a collective choice rule. There are two obstacles to such a hasty conclusion, however.

The first obstacle is that, following Debreu (1972), each individual $i$ 's preferences in Chichilnisky and Heal (1983) are represented by a locally integrable $C^{1}$ normalized gradient field $\mathbf{v}_{i}$ over the choice set, which is typically modeled as the unit ball in an Euclidean space. When the space of admissible preferences is convex, a natural candidate for an aggregation

\footnotetext{
${ }^{6}$ The surveys by Lauwers (2000, 2009) and Baigent (2011) offer useful discussions of these axioms.
} 
procedure is simply to take the average of the vector fields $\mathbf{v}_{i}, i=1, \ldots, I$; this construction easily extends to the case where the space of admissible preferences is a retract of its convex hull. However, these operations typically do not preserve the convexity of preferences, as required by (6), and are thus not adapted to the present framework.

The second obstacle is that the space of admissible preferences in Chichilnisky and Heal's (1983) characterization result is assumed to have a particular topological structure; namely, that it is a path-connected parafinite $C W$ complex. $^{7}$ Roughly speaking, this means that the preference space is built in a countable number of stages, each stage being obtained from the previous one by adding cells of a given finite dimension. Horwath (2001) extends this result to a much broader class of admissible preferences, but it is not straightforward to determine whether the space $\mathbf{P}_{v, d s c}$ belongs to that class.

The restrictions imposed by Chichilnisky and Heal (1983, Theorem 1) to the preference space, therefore, prevent us from directly applying their characterization result. Fortunately, the explicit construction of the contraction $h_{\bar{u}}$ of $\mathbf{U}_{v, d s c}$ into any given $\bar{u} \in \mathbf{U}_{v, d s c}$ provided in Theorem 1 allows us to exhibit a collective choice rule that satisfies the desired properties. As in the proof of Theorem 1, we can identify preferences in $\mathbf{P}_{v, d s c}$ with their representations in $\mathbf{U}_{v, d s c}$. The following result then holds.

Theorem 2 Define inductively a sequence of collective choice rules as follows: for each $u_{1} \in \mathbf{U}_{v, d s c}$, let

$$
\Phi_{1}\left(u_{1}\right) \equiv u_{1}
$$

and for all $I \geq 2$ and $\left(u_{1}, \ldots, u_{I}\right) \in \mathbf{U}_{v, d s c}^{I}$, let

$$
\Phi_{I}\left(u_{1}, \ldots, u_{I}\right) \equiv h_{\Phi_{I-1}\left(u_{1}, \ldots, u_{I-1}\right)}\left(u_{I}, \frac{I-1}{I}\right)
$$

Then, for each $I \geq 1, \Phi_{I}: \mathbf{U}_{v, d s c}^{I} \rightarrow \mathbf{U}_{v, d s c}$ satisfies Anonymity, Unanimity, and Continuity.

Proof. Proceeding along the lines of the proof of Theorem 1, a straightforward induction shows that $\Phi_{I}\left(u_{1}, \ldots, u_{I}\right) \in \mathbf{U}_{v, d s c}$ for all $I$ and $\left(u_{1}, \ldots, u_{I}\right) \in \mathbf{U}_{v, d s c}^{I}$. We now check each axiom in turn.

(Anonymity) We prove that for each $I$, the transformation $\Phi_{I}$ associates to each $t^{\prime}$ such that $\left(0_{\ell}, t^{\prime}\right) \in V$ and to each profile $\left(u_{1}, \ldots, u_{I}\right) \in \mathbf{U}_{v, d s c}^{I}$ an indifference set that is the vertical convex combination of the indifference sets $u_{1}^{-1}\left(\left\{t^{\prime}\right\}\right), \ldots, u_{I}^{-1}\left(\left\{t^{\prime}\right\}\right)$ with identical weights

\footnotetext{
${ }^{7}$ See Spanier (1966) for precise definitions of these terms.
} 
$1 / I$, bearing in mind that, by normalization, $u_{i}\left(0_{\ell}, t^{\prime}\right)=t^{\prime}$ for all $i=1, \ldots, I$. That $\Phi_{I}$ is anonymous then follows from the symmetry of this procedure. The proof is by induction. First, note that the result trivially holds for $\Phi_{1}$. Let then $I \geq 2$, and suppose that the result holds for $\Phi_{I-1}$. Then, according to the construction of the homotopy $h_{\Phi_{I-1}\left(u_{1}, \ldots, u_{I-1}\right)}$ provided in the proof of Theorem $1, \Phi_{I}$ associates to each $t^{\prime}$ such that $\left(0_{\ell}, t^{\prime}\right) \in V$ and to each profile $\left(u_{1}, \ldots, u_{I}\right) \in \mathbf{U}_{v, d s c}^{I}$ an indifference set that is the vertical convex combination of the indifference sets $\left(\Phi_{I-1}\left(u_{1}^{-1}\left(\left\{t^{\prime}\right\}\right), \ldots, u_{I-1}\right)\right)^{-1}\left(\left\{t^{\prime}\right\}\right)$ and $u_{I}^{-1}\left(\left\{t^{\prime}\right\}\right)$ with weights $(I-1) / I$ and $1 / I$. The desired implication then follows from the induction hypothesis.

(Unanimity) The proof is again by induction. First, note that the result trivially holds for $\Phi_{1}$. Let then $I \geq 2$, and suppose that the result holds for $\Phi_{I-1}$. Then, for each $u \in \mathbf{U}_{v, d s c}$, we have $\Phi_{I}(u, \ldots, u)=h_{\Phi_{I-1}(u, \ldots, u)}(u,(I-1) / I)=h_{u}(u,(I-1) / I)=u$ by construction of the homotopy $h_{u}$, as desired.

(Continuity) The proof is yet again by induction. First, note that the result trivially holds for $\Phi_{1}$. Let then $I \geq 2$, and suppose that the result holds for $\Phi_{I-1}$. To complete the induction step, we need to show that, for $\xi \equiv(I-1) / I$, the mapping $H: \mathbf{U}_{v, d s c} \times \mathbf{U}_{v, d s c} \rightarrow$ $\mathbf{U}_{v, d s c}:(\bar{u}, u) \mapsto h_{\bar{u}}(u, \xi)$ is continuous. The proof is similar to the contractibility argument in Theorem 1 and is, therefore, omitted. Hence the result.

Observe again that the compensation principle expressed by A6 plays a key role in the proof of Theorem 2. By contrast, consider what happens when A6 is violated by each individual preference relation in a profile, as illustrated in Figure 4 below.

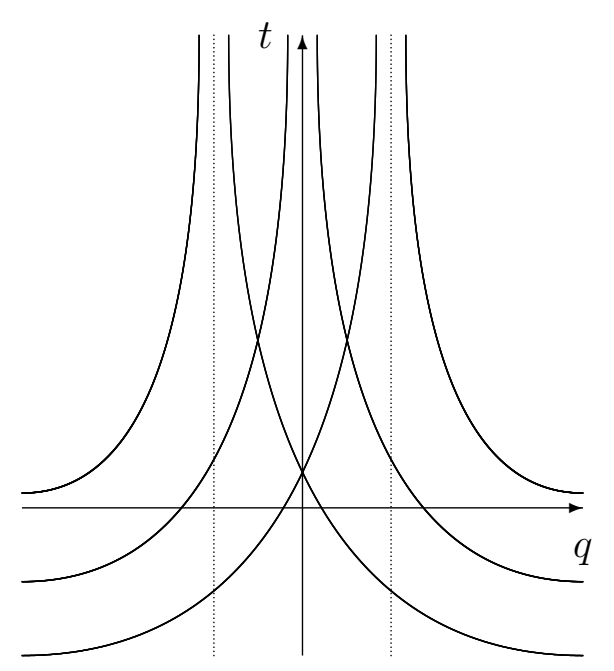

Figure 4 Two individual preference relations that violate A6. 
The two individuals whose preferences are depicted in Figure 3 have opposite interests in the $q$ dimension, and transfers in the $t$ dimension are not effective enough to compensate either of them for holding large (in absolute value) undesired amounts of $q$. It is then unclear how to aggregate their preferences into a collective preference relation that satisfies, in particular, strict convexity. ${ }^{8}$ It is thus an open question whether the space $\mathbf{P}_{d s c}$ of preferences that satisfy A1-A5 and A7-A8, but not necessarily A6, admits a collective choice rule that is continuous, anonymous, and respects unanimity.

\footnotetext{
${ }^{8}$ Note in that respect that the additive rule for normalized gradient fields used by Chichilnisky (1980) and Chichilnisky and Heal (1983) will not do. Suppose for instance that individual preferences are represented by $u_{1}(q, t)=-q-1 /(t+1)$ and $u_{2}(q, t)=q-1 /(t+1)$ for $(q, t) \in V \equiv \mathbb{R} \times(-1, \infty)$, so that indifference sets are convex hyperbolas. Then the addition of the normalized gradients of $u_{1}$ and $u_{2}$ is everywhere equal to $(0,1)$, which does not correspond to strictly convex preferences: the candidate collective indifference sets are flat, reflecting that, at each point of $V$, individuals 1 and 2 only agree along the $t$ dimension.
} 


\section{References}

[1] Arrow, K.J. (1951) Social Choice and Individual Values. New York: John Wiley \& Sons.

[2] Baigent, N. (2011): "Topological Theories of Social Choice," in Handbook of Social Choice and Welfare, ed. by K. Arrow, A. Sen, and K. Suzumura. Amsterdam: Elsevier, 301-334.

[3] Becker, G.S. (1981): "Altruism in the Family and Selfishness in the Market Place," Economica, 48(189), 1-15.

[4] Chiappori, P.-A. (1988): "Rational Household Labor Supply," Econometrica, 56(1), 6390.

[5] Chiappori, P.-A. (1992): "Collective Labor Supply and Welfare," Journal of Political Economy, 100(3), 437-467.

[6] Chichilnisky, G. (1980): "Social Choice and the Topology of Spaces of Preferences," Advances in Mathematics, 37(2), 165-176

[7] Chichilnisky, G., and G. Heal (1983): "Necessary and Sufficient Conditions for a Resolution of the Social Choice Paradox," Journal of Economic Theory, 31(1), 68-87.

[8] Debreu, G. (1972): "Smooth Preferences," Econometrica, 40(4), 603-615.

[9] Horvath, C.D. (2001): "On the Topological Social Choice Problem," Social Choice and Welfare, 18(2), 227-250.

[10] Kannai, Y. (1970): "Continuity Properties of the Core of a Market," Econometrica, $38(6), 791-815$.

[11] Klee, V.L., Jr. (1953): "Convex Bodies and Periodic Homeomorphisms in Hilbert Space," Transactions of the American Mathematical Society, 74(1), 10-43.

[12] Lauwers, L. (2000): "Topological Social Choice," Mathematical Social Sciences, 40(1), $1-39$.

[13] Lauwers, L. (2009): "The Topological Approach to the Aggregation of Preferences," $33(3), 449-476$.

[14] Mas-Colell, A. (1985): The Theory of General Economic Equilibrium: A Differentiable Approach, Cambridge, UK: Cambridge University Press. 
[15] Mas-Colell, A., M.D. Whinston, and J.R Green (1995): Microeconomic Theory, Oxford: Oxford University Press.

[16] Polemarchakis, H.M., and P. Siconolfi (1993): "Competitive Equilibria Without Free Disposal or Nonsatiation," Journal of Mathematical Economics, 22(1), 85-99.

[17] Spanier, E.H. (1966): Algebraic Topology, New York: McGraw-Hill.

[18] Varian, H.R. (1992): Microeconomic Analysis, Third Edition, New York: W.W. Norton \& Company, Inc.

[19] Wold, H., and L. Juréen (1953): Demand Analysis: A Study in Econometrics, New York: John Wiley \& Sons, Inc. 